\title{
High dimensional parameter tuning for event generators
}

\author{
Johannes Bellm $^{\mathrm{a}}$, Leif Gellersen ${ }^{\mathrm{b}}$ \\ Department of Astronomy and Theoretical Physics, Lund University, 22362 Lund, Sweden
}

Received: 25 September 2019 / Accepted: 20 December 2019 / Published online: 23 January 2020

(C) The Author(s) 2020

\begin{abstract}
Monte Carlo Event Generators are important tools for the understanding of physics at particle colliders like the LHC. In order to best predict a wide variety of observables, the optimization of parameters in the Event Generators based on precision data is crucial. However, the simultaneous optimization of many parameters is computationally challenging. We present an algorithm that allows to tune Monte Carlo Event Generators for high dimensional parameter spaces. To achieve this we first split the parameter space algorithmically in subspaces and perform a Professor tuning on the subspaces with binwise weights to enhance the influence of relevant observables. We test the algorithm in ideal conditions and in real life examples including tuning of the event generators Herwig 7 and Pythia 8 for LEP observables. Further, we tune parts of the Herwig 7 event generator with the Lund string model.
\end{abstract}

\section{Introduction and motivation}

The amount of data taken at the LHC allows measuring observables that can be calculated perturbatively to high precision. This is beneficial for the comparison as well as the improvement of phenomenologically motivated nonperturbative models and also the searches for new physics. With the increasing precision made available in recent years through perturbative higher order calculations, theoretical uncertainties have reduced dramatically. In the comparison of these theory predictions and experimental data, Monte Carlo event generators (MCEG) [1] like Herwig 7 [2-5], Sherpa [6] or Pythia 8 [7,8] play an important role. If possible a matched calculation that includes the perturbative corrections and the effects described by the MCEG can give an improved picture of the event structure measured by the experiment.

\footnotetext{
a e-mail: johannes.bellm@thep.lu.se

b e-mail: leif.gellersen@thep.lu.se
}

Here event generators typically include additional phenomenological models to include effects that are not part of specialised fixed order and resummed calculations. Uncertainties of these additional modelled, but factorised, parts of the simulation can be estimated from lower order simulations. The MCEG contain various, usually factorized (e.g. by energy scales) components. In the development, these parts can be improved individually. The aforementioned matching to perturbative calculations is an example of recombining parts usually separated in the event generation, namely the parton shower and hard matrix element calculation. While it is possible to make such modifications and improvements, it is also necessary to keep other parts of the simulation in mind. Even though the generation is factorized, various parts of the simulation will have an impact on other ingredients of the generator. Any modification can, in general, have an impact on the full events. Calculated, or at least theoretically motived improvements will lead to a reduction of freedom that eventually also restricts the parameter ranges of the phenomenological models that could be used to compensate the variations of the perturbative side [9-16]. The capability to describe data needs to be reviewed with the modifications made in order to use the event generator for future predictions or concept designs for new experiments.

The procedure of adjusting the parameters of the simulation to measured data is called tuning. Various contributions for the tuning of MCEGs have been made [17-25], and the importance of these studies can be deduced from the recognition received. More recently, new techniques have been presented that can improve the performance of tuning [2630]. To be able to perform the comparison of simulation and data, the data needs to be collected and it needs to be possible to analyse the simulations similar to the experimental setup. Here, the hepdata project [31] and analysis programs like Rivet [32] are of great importance to the high energy physics community. Once the data and the possibility to analyse is given, the 'art' of tuning is to choose the 'right' data, possibly enhance the importance of some data sets over 
others, and to modify the parameters of the simulation such to reduce the difference of data and simulation. A prominent tool to allow the experienced physicist to perform the tuning is the Professor [21] package that allows performing most of the procedure automatically.

The complexity of the MCEG tuning depends on the dimension of the parameter space used as an input to the event generation. Further, the measured observables are in general functions of many of the parameters used in the simulation. In this contribution, we address the problems of high dimensional parameter determination. We propose a method to choose subsets of parameters to reduce the complexity. We further aim to automatize the tuning process, to be able to retune with minimal effort once improvement is made to the MCEG in use. We call this automation of the tuning process and the algorithm to perform it the Autotunes method. ${ }^{1}$ As possible real life scenarios we then tune the Herwig 7 and Pythia 8 models and also a hybrid form, namely the Herwig 7 showers with the Pythia 8's Lund String model $[33,34]$.

We structure the paper as follows: In Sect. 2 we define the problem and questions that we want to solve and answer. We then describe Professor and its the capabilities and restrictions. In Sect. 3 we explicitly define the algorithm and point out how the methods used will act mathematically. In Sect. 4 we show how the algorithm was tested. Results of tuning the event generators Herwig 7 and Pythia 8 are presented in Sect. 5. We conclude in Sect. 6 and specify the possible next steps.

\section{Current state}

Improving the choice of parameters - commonly referred to as tuning - is required to produce the most reliable theory predictions. The Rivet toolkit allows comparing Monte Carlo event generator output to data from a variety of physics analyses. Based on this input, different tuning approaches can be followed. A most elaborate approach is the tuning 'by hand'. It requires a thorough understanding of the physical processes involved in the generation of events and the identification of suitable observables to adjust every single parameter. A detailed example of such a manual approach is given by the Monash tune [22], the current default tune of the event generator Pythia $8[7,8]$. However, in order to simplify and systematize tuning efforts, a more automated approach is desirable. The Professor [21] tuning tool was developed for this purpose. This allows to tune multiple parameters simultaneously.

\footnotetext{
${ }^{1}$ An implementation of the method will be made available on: https://gitlab.com/Autotunes.
}

\subsection{Professor: capabilities and restrictions}

The Professor method of systematic generator tuning is described in detail in [21]. The basic idea is to define a goodness of fit function between data generated with a Monte Carlo event generator and reference data that is provided by experimental measurements through Rivet. This function is then minimized. Due to the high computational cost of generating events, a direct evaluation of the generator response in the goodness of fit function should be avoided. This is done by using a parametrization function, usually a polynomial, which is fitted to the generator response to give an interpolation which allows for efficient minimization. The following $\chi^{2}$ measure is used as a goodness of fit function between each bin $i$ of observables $\mathcal{O}$ as predicted by the Monte Carlo generator $f_{i}$, depending on the chosen parameter vector $\vec{p}$ and as given by the reference data $\mathcal{R}_{i}$. To simplify the notation, each bin in each histogram is now - without loosing generality called an observable, with prediction $f_{i}$ and reference data value $\mathcal{R}_{i}$ :

$\chi^{2}(\vec{p})=\sum_{i} w_{i} \frac{\left(f_{i}(\vec{p})-\mathcal{R}_{i}\right)^{2}}{\Delta_{i}^{2}}$

The uncertainty of the reference observable is denoted by $\Delta_{i}$. Furthermore, a weight $w_{i}$ is introduced for every observable. These weights can be chosen arbitrarily to bias the influence of each observable in the tuning process.

The approach of the Professor method allows to tune multiple parameters simultaneously, and drastically reduces the time needed to perform a tune. The number of parameters and the polynomial approximation of the generator response limits the efficiency for a high number of parameters or a high degree of polynomial power. The formula is given in [21] and already a set of 15 parameters and an approximation using third power requires at least 816 generator samples to form an interpolation. To test the stability of such interpolations the method of runcombinations can be used to check how well the minimization is performed. Here a higher number than the minimal set of points is needed. However, further effort is needed to overcome some of the restrictions that remain:

- The polynomial approximation of the generator response is well suited for up to about ten parameters. Further simultaneous tuning requires many parameter points as input for the polynomial fit, typically exceeding the available computing resources. This is often circumvented by identifying a subset of correlated parameters ${ }^{2}$ that should be tuned simultaneously.

\footnotetext{
${ }^{2}$ Here and in the following we use the term correlated parameters in the sense to influence same observables. We do not discriminate between correlation or anti-correlation.
} 
- The assignment of weights requires the identification of relevant observables for the set of parameters. Different choices and methods can possibly bias the tuning result.

- Correlations in the data need to be identified in order to reduce the weight of equivalent data in the tune, and thus avoid bias by over-represented data.

- The polynomial approach is reasonable in sufficiently small intervals in the parameters, but might fail if the initial ranges for the sampled parameters are chosen too large and the parameter variation shows a nonpolynomial behaviour. ${ }^{3}$

\subsection{Suggested improvements}

In the Autotunes approach we aim to address some of the issues mentioned above. For high-dimensional problems, we suggest a generic way to identify correlated high-impact parameters that need to be tuned simultaneously, and divide the problem into suitable subsets. Instead of setting weights for every observable by hand, we propose an automatic method that sets a high weight on highly influential observables for every sub-tune, reducing the bias by observables that are better optimized by parameters in another sub-tune. This procedure makes the tuning process more easily reproducible.

As a further improvement, we implement an automated iteration of the tuning process, that takes refined ranges from the preceding tune as a starting point. By a stepwise reduction of the parameter ranges, we improve the stability and reliability of our first order approximation of parameter impact, and the polynomial interpolation implemented in Professor.

\section{The algorithm}

In this section we formulate the algorithm proposed to improve the tuning of the high dimensional parameter space. We propose to organize the algorithm as:

(A) Reduce the dimensionality of the problem by splitting the parameters into subsets, defining sub-spaces and sub-tunes. Here the algorithm should cluster parameters that are correlated.

(B) Assign weights to observables, such that the current sub-tune predominantly acts to reduce the weighted $\chi^{2}$ calculation for the corresponding sub-space.

(C) Run Professor on the sub-tunes.

\footnotetext{
${ }^{3}$ For the method it would be beneficial to reformulate the theoretical model such that the parameter response on typical experimental observables is of polynomial type. For general observables and event generators such a reformulation is in general not given. Additional work to identify such behaviour could be worth pursuing.
}

(D) Automatically find new parameter ranges for an iterative tuning.

\subsection{Reduce the dimensionality (chunking)}

The goal of this step is to split up a high dimensional space $(N$ dimensional) into subspaces ( $n$ dimensional ${ }^{4}$ ), such that the clustered parameters are correlated on the observable level. To achieve this we have to define a quantity $\mathcal{M}$ that can be maximized or minimized to allow the algorithmic treatment. The parameter space we work with is a hyper-rectangle. The observable definitions usually allow to access one dimensional projections. Here, the 'projection' is the model (implemented in an event generator) at hand.

Two issues directly come to mind: First, we explicitly describe the parameter space $\vec{p} \in\left[\vec{p}_{\min }, \vec{p}_{\text {max }}\right]$ as a hyperrectangle rather than a hyper-cube. Some of the parameters could have been measured externally, others are pure model specific. A measure, which allows comparisons between the parameters, needs to be corrected for the initial ranges ( $\left.\left[\vec{p}_{\min }, \vec{p}_{\max }\right]\right)$ defined by the input. To overcome this first problem, we first define $\bar{p}^{\alpha} \in[0,1]$ as the vector ${ }^{5}$ normalized to the input range and will describe below how a rescaling is performed to regain the information lost by this normalisation and relate it to the variations on the observables.

The second issue is the generic observable definition. Some of the observable bins are parts of normalized distributions, or even related to other histograms (as is the case for e.g. centrality definitions in heavy ion collisions [35]). In other words, the height $f_{i}$ of observables again does not define a good measure to define a generic quantity to minimize. In order to overcome the second problem, we test the observable space with $N_{\text {search }}$ random points in the parameter space projected with the model to the observables. The spread for each observable is used to normalize the values to $\bar{f}_{i} \in[0,1]$. Note that an influential parameter can be shadowed by a less important parameter if the latter has a too large initial range. After the normalizations $\bar{p}^{\alpha}$ and $\bar{f}_{i}$ are performed, we use the $N_{\text {search-projections to perform linear regression fits for }}$ each parameter, and for each observable bin. Here, the linear dependence defines the slope of each parameter in each bin $d \bar{f}_{i} / d \bar{p}^{\alpha}$. Due to the normalization of the $f_{i}$-range, this slope is influenced not only by the parameter itself, but also by the spread produced by the other parameters. The reduction of the slope includes a correlation of parameters to other parameters on the observable level. We use the absolute value ${ }^{6}$ of

\footnotetext{
${ }^{4}$ Here, the dimension $n$ is chosen such that the Professor package can easily manage the given subspace.

5 We use Greek letters to distinguish vector indices in the parameter space from Latin letter indices used for observables bins.

${ }^{6}$ The later normalization of $\overrightarrow{\mathcal{S}}_{i}$ but also the later definition of $\mathcal{M}$ requires the absolute value.
} 
the slope to define an averaged gradient or slope-vector $\overrightarrow{\mathcal{S}}_{i}$. The sum $\overrightarrow{\mathcal{S}}_{\text {Sum }}=\sum_{i} \overrightarrow{\mathcal{S}}_{i}$ has in general unequal entries, one for each parameter in the tune. This indicates that the input ranges $\left[\vec{p}_{\min }, \vec{p}_{\max }\right]$ are of unequal influence on the observables. To correct for this choice and to improve the clustering of parameters with higher correlation, we normalize each $\overrightarrow{\mathcal{S}}_{i}$ element-wise with $\overrightarrow{\mathcal{S}}_{\text {Sum }}$ to create $\overrightarrow{\mathcal{N}}_{i}$,

$\mathcal{N}_{i}^{\alpha}=\frac{\mathcal{S}_{i}^{\alpha}}{\mathcal{S}_{\text {Sum }}^{\alpha}}$.

To illustrate the effect of the element-wise normalisation we show in Fig. 1 how the sum of all normalised vectors of the observable bins (here 3 ) reaches the parameter-space point $(1)^{N}$ ( here $N=2$ ). In bin $i$ the component to a parameter $\alpha$ of the new vector $\overrightarrow{\mathcal{N}}_{i}$ is reduced if other observables are sensitive to the same parameter. The direction of $\overrightarrow{\mathcal{N}}_{i}$ indicates the correlation of parameters. We can now use $\overrightarrow{\mathcal{N}}_{i}$ to chunk the dimensionality of the problem. ${ }^{7}$ Therefore, we calculate the projection for each of the $\overrightarrow{\mathcal{N}}_{i}$ on all possible $n$ dimensional sub-spaces. This is done by multiplication with combination vectors $\overrightarrow{\mathcal{J}}$. Here, $\overrightarrow{\mathcal{J}}$ is defined as one of all possible $N$-dimensional vectors with $N-n$ zero entries and $n$ unit entries, where $n$ is again the dimension of the desired subspace, e.g. $\overrightarrow{\mathcal{J}}=(1,0,0, \ldots, 1,0,1)$. The sub-space then defines a sub-tune. The sum over all projections,

$$
\sum_{i}\left(\overrightarrow{\mathcal{N}}_{i} \cdot \overrightarrow{\mathcal{J}}\right)^{k}
$$

can serve as a good measure to be maximized. However, due to the normalization of $\overrightarrow{\mathcal{N}}_{i}$ the sum is equal $\overrightarrow{\mathcal{J}}$ for $k=1$. For the quantity $\mathcal{M}$ mentioned at the start of the section we use $k=2$ giving,

$\mathcal{M}(\overrightarrow{\mathcal{J}})=\sum_{i}\left(\overrightarrow{\mathcal{N}}_{i} \cdot \overrightarrow{\mathcal{J}}\right)^{2}$

in order to define the sub-tunes. This choice of $k>1$ selects a few strongly correlated parameters over many less correlated ones. The maximal $\mathcal{M}\left(\overrightarrow{\mathcal{J}}_{\text {Step } 1}\right)$ defines the first of the sub-tunes (Step 1). For other steps, we require no overlap between the sub-spaces. This we enforce by requiring a vanishing scalar product $\overrightarrow{\mathcal{J}}_{\text {StepN }} \cdot \overrightarrow{\mathcal{J}}_{\text {StepM }}$. It is now possible to perform the tuning in the same order as the maximal measures of Eq. (4) are found. This would first fix parameters that can modify the description of fewer observables, and

\footnotetext{
7 A linear regression used is blind to more complicated parameter dependence. However, we assume at this point that on the one hand there is more than one bin that is important to a given parameter, and on the other hand a strong variation of the parameter with a small slope will reduce the importance of the bin in this iteration and will become more important in the next iteration if the parameter range is shifted and new slopes are calculated.
}

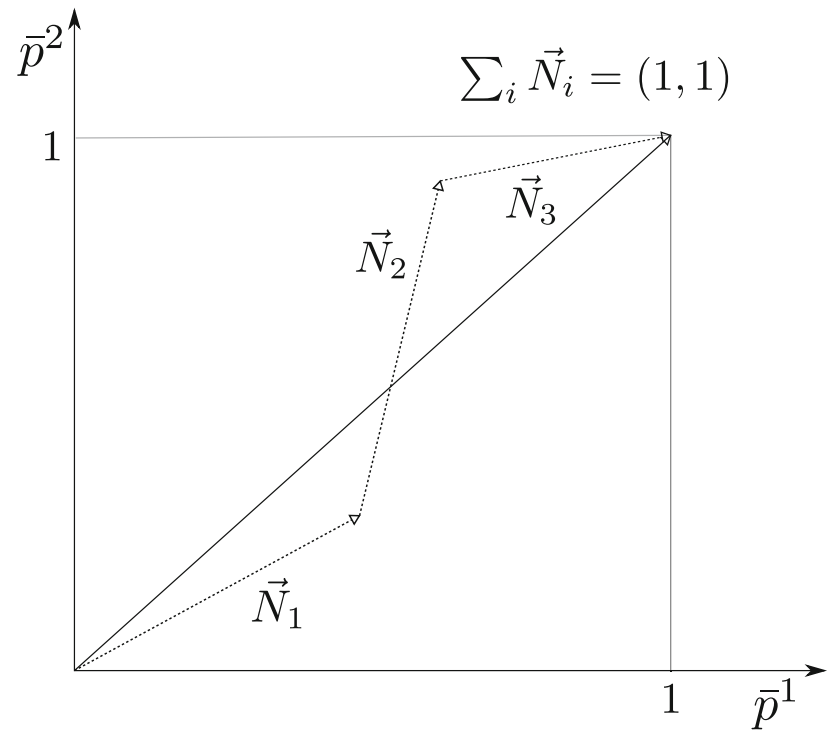

Fig. 1 Illustration of a two-dimensional parameter space and the normalized slope vectors that sum up to reach the all-one position of a hyper-cube. Projections of the individual vectors help to split up the parameter space and to assign weights to the sub-tunes

then continue to vary parameters that are globally important. In order to first constrain globally important parameters, and then fix specialized parameters, we invert the order of found sub-tunes. We thus have split the dimensionality of the problem, and will ensure, in the following, that observables used in the various sub-tunes are described by the set of influential parameters.

\subsection{Assign weights (improved importance)}

In the last paragraph, we described how we split up the dimensionality of the full parameter set to allow us to tune subsets, such that parameters with higher correlation on the observable level are tuned simultaneously. To increase the importance of observables that are relevant for the sub-tune, we now try to enhance the relative weight with respect to other observables. Here, we use the same vectors $\overrightarrow{\mathcal{N}}_{i}$ defined in the last paragraph. These vectors, obtained by linear regression, and normalized to the overall range of observable vectors have the properties, that they point in the parameter space, and, due to the normalization, they correlate the importance of other measured observables to the current bin. We define the weight of the observable bins later used to minimize the $\chi^{2}$ as

$w_{i}=\frac{\left(\overrightarrow{\mathcal{N}}_{i} \cdot \overrightarrow{\mathcal{J}}_{\text {Step }}\right)^{2}}{\sum_{\alpha} N_{i}^{\alpha}}$,

where $\overrightarrow{\mathcal{J}}_{\text {Step }}$ is the combinatorial vector defined in Sect. 3.1, corresponding to the sub-tune. This weight has the properties 
that the multiplication in the numerator increases the weight of the important bins for the sub-tune, while the sum over components of $\vec{N}_{i}$ in the denominator reduces the importance of bins that are equally or more important to other parameters. Note that the $\vec{N}_{i}$ itself are not normalised, only the sum over $i$ is normalised in each component.

This weighting enhances the effect of bins that have been identified as influential with respect to the parameters tuned in the given sub-tune. It also reduces the effect of bins that are expected to be relevant in other sub-tunes. Thereby, the algorithm reduces bias by not yet optimized data bins and less relevant distributions. This weighting is applied for each tune step individually and does not take into account the physicists knowledge of relevant or unsuitable data from a physics perspective. An additional global weighting based on physical motivations can be performed on top of this algorithmic treatment, but is not considered in this work.

We want to highlight that the splitting of the parameter space, described in Sect. 3.1 as well as the assignment of weights described here are blind to the data measured in the experiment. Only the generator response on the defined observables are needed.

\subsection{Run Professor (tune-steps)}

Before we start the first iteration and step, we perform a second order Professor tune as starting condition, referenced to as BestGuessTune. Here, we make use of the $N_{\text {search }}$ sampled points used to determine the splitting of the parameter space and the weight setting described in the previous sections. Instead of giving ranges and a starting parameter point, only ranges are required as starting conditions. As the starting point has an impact on the sub-tunes that are performed in the beginning, the BestGuessTune aims to reduce user interference.

After splitting the parameter space and enhancing the weights for important observables for the sub-tunes we use the capability of Professor to tune the parameter space of each step. When a step is performed, we use the Professor result of this and all previous steps to fix the parameters for the following step.

For the individual sub-tunes, we make use of the runcombination method of Professor, to build subsets of the randomly sampled parameter points. This produces modified polynomial interpolations and gives a spread in the $\chi^{2}$ values of the best fit values. We choose the result associated with the best $\chi^{2}$ as the best tune value. To give a measure for the stability of the tune, we choose the runcombinations that give the best $80 \%$ of the $\chi^{2}$ values. For those we extract the corresponding parameter range, and add a $20 \%$ margin on both sides. To elucidate the effect, an example for the tuning of the strong coupling constant $\alpha_{\mathrm{S}}$ is given in Fig. 2. Here, the blue points correspond to the
$80 \%$ best combinations and the green dashed lines give the measure of stability. Diagrams like Fig. 2 are automatically produced by the program, for each parameter and tune-step. In Fig. 2 three iterations are shown as it is described in the next section.

\subsection{Find new ranges and iterate the procedure (Iteration)}

The measure of stability defined in the Sect. 3.3 also serves as input for the next iterations. Here we make use of the redefined ranges. An iterative tuning is important, since the first set of parameters has been influenced by the users choices, and a next iteration can have significant impact on the parameter value. For very expensive simulations, at least a retuning of the first step's parameter space seems desirable. The program is setup such that one can use the output of the first full tune as input for the next iteration.

\section{Testing and findings}

Before applying the Autotunes framework to perform a LEP retune of Pythia 8, Herwig 7, and a combination of both in Sect. 5, we test the method under idealized conditions. First, we tune the coefficients of a set of polynomials. The observables used for the tune are constructed from the polynomials for a random choice of coefficients, see Sect. 4.1. As a second test, we tune the Pythia 8 event generator to pseudo data generated with randomized parameter values. In both scenarios, it is desirable to recover the randomly chosen parameter values that were used to generate the observables.

\subsection{Testing the algorithm under ideal conditions}

To test the algorithm, we first introduce a simplified and fast generator. We define the projection,

$$
\begin{aligned}
\mathcal{O}_{a}= & G_{0, a}+G_{1, a}^{i} C_{a}^{i r} p^{r}+G_{2, a}^{i j} C_{a}^{i r} p^{r} p^{j} \\
& +G_{3, a}^{i j k} C_{a}^{i r} p^{r} p^{j} p^{k}+G_{4, a}^{i j k l} C_{a}^{i r} p^{r} p^{j} p^{k} p^{l},
\end{aligned}
$$

with $m$-dimensional tensors $G_{m, a}^{\cdots}$, correlation matrices ${ }^{8} C_{a}^{i r}$, and parameter points $p^{i}$. Upper indices sum over the parameter dimensions. We fill $G_{m}^{\cdots}$ with random numbers and use $C_{a}^{i r}$ to correlate subsets of parameters. Here $C_{a}^{i r}$ is a diagonal matrix with constant entries $k>1$ if the bin $a$ should be enhanced for this parameter $i$ and one if not. By building ranges, we can define enhanced parameter sets. As an example, we use a $d=15$ dimensional parameter space,

\footnotetext{
8 As mentioned before, we understand the correlation of parameters only on the level of influencing similar observables. It is therefore a simple choice to enhance subsets of parameters in the way described without off-diagonal entries in the correlation matrices.
} 

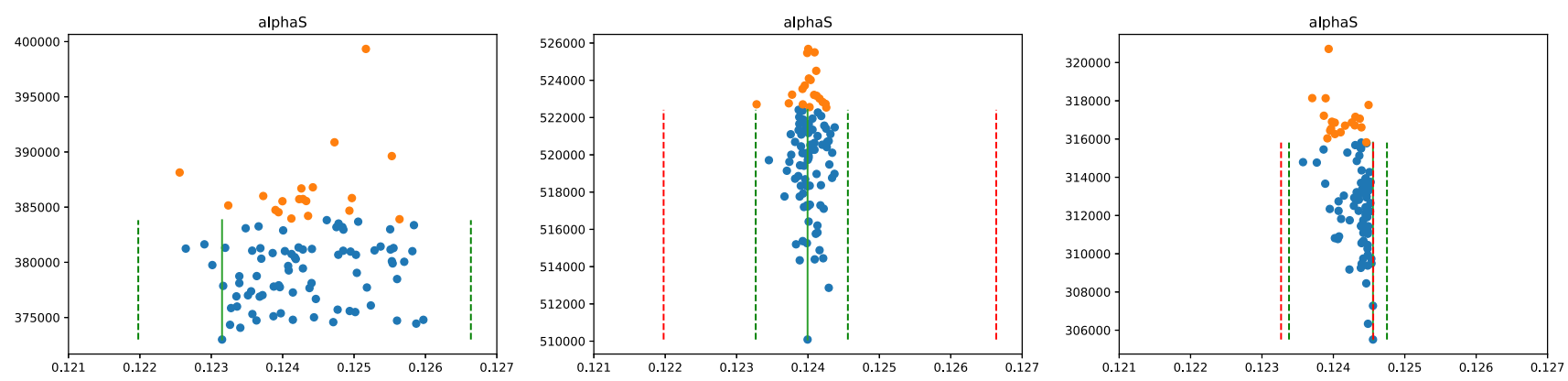

Fig. 2 Example of tune results for the $\alpha_{S}\left(M_{Z}\right)$ value with three iterations from left to right. The points are Goodness of fit (G.o.F.) values given by Professor for various runcombinations (see Sects. 3.3 and 3.4 for details). While the old parameter ranges are given by dashed red lines, the $80 \%$ of the best G.o.F. values (blue points) determine the new green ranges for the next iteration. The $20 \%$ of the worst G.o.F. values

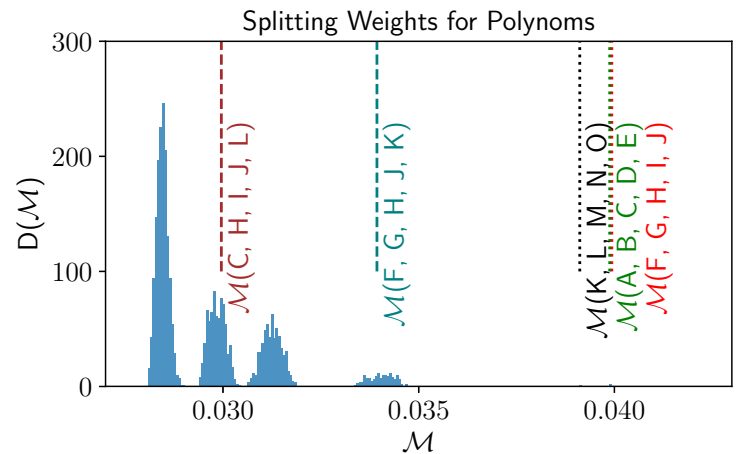

(orange points) are neglected. The lowest G.o.F. value (solid green line) defines the current best tune value that is used as starting value for next tune-steps and iterations. The G.o.F. values are calculated including the weights for the observables given by the algorithm. Hence, for the best fit and the range only the relative values are important and G.o.F. values between iterations are not comparable

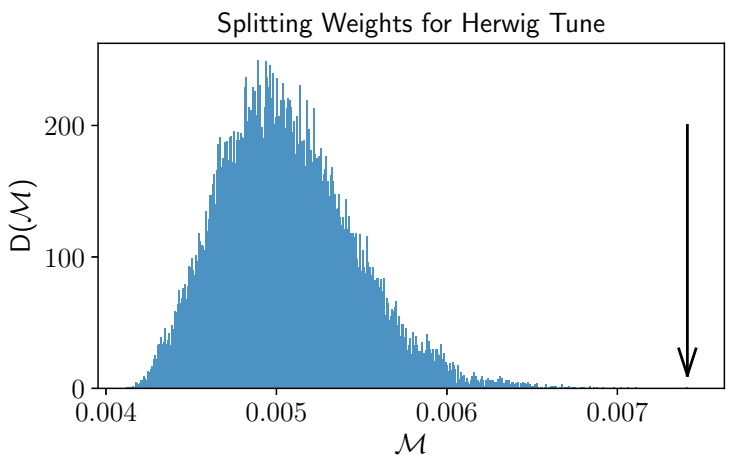

Fig. 3 Left: measure density for all combination vectors according to Eq. (4) for ideal conditions of polynomial functions, see Sect. 4.1. Right: measure density for MCEG tuning (here Herwig angular ordered shower with cluster model). Although the distribution is less resolving, a structure is visible

and correlate the parameter in combinations as $[\mathrm{A}, \mathrm{B}, \mathrm{C}, \mathrm{D}$, $\mathrm{E}],[\mathrm{F}, \mathrm{G}, \mathrm{H}, \mathrm{I}, \mathrm{J}]$ and $[\mathrm{K}, \mathrm{L}, \mathrm{M}, \mathrm{N}, \mathrm{O}]$. Under these ideal conditions, we search for the correlations with the procedure described in Sect. 3.1. In Fig. 3(left), the weights for the parameter correlations are shown. The ideal combinations defined above create the highest weights, and would therefore be detected as correlated by the algorithm. In a real life MCEG tune the correlations are much less pronounced. In the right panel of Fig. 3, we show the weight distribution for the example of the Herwig 7 tune described in Sect. 5.2.1. Once the correlated combinations are found, the algorithm continues with the procedure described in Sects. 3.3 and 3.2. As the result of each full tune serving as input to a next iteration, it is possible to visualize the outcome as a function of tune iterations. Figure 4 shows this visualisation as produced by the program. Each parameter $(\mathrm{A}-\mathrm{O})$ is normalised to the initial range, and plotted with an offset. In this example, it is possible to show the input values of the pseudo data with dashed lines. This is not possible when tuning is performed to real data. As Professor is very well capable of finding polynomial behaviour, the parameter point that the method aims to find is already well constrained after the first itera- tion. However, next iterations still improve the result. This may be seen for example in the third and last line.

The procedure to split the parameter space into smaller subsets, and to assign weights can suffer from numerical and statistical noise if we consider many observables. In Appendix A, we discuss the range dependence and show that the weight distributions are fairly stable if the same parameters are found to be correlated. It is further possible to ask for weights, if all parameters should be tuned independently. From the tuning perspective this seems an unnecessary feature, but can help to find observables that are likely influenced by a model parameter, e.g it is possible to identify the range of bins where the bottom mass has influence in jet rates.

\subsection{Tuning Pythia 8 to pseudo data}

As a second test of our method, we use Pythia 8 to generate pseudo data for a random choice of 18 relevant parameter values. We then use three different methods to tune Pythia 8 to this set of pseudo data, and try to recover the true parameters. In all methods, we divide the tuning into three subtunes. The first method is a random selection of parameters 


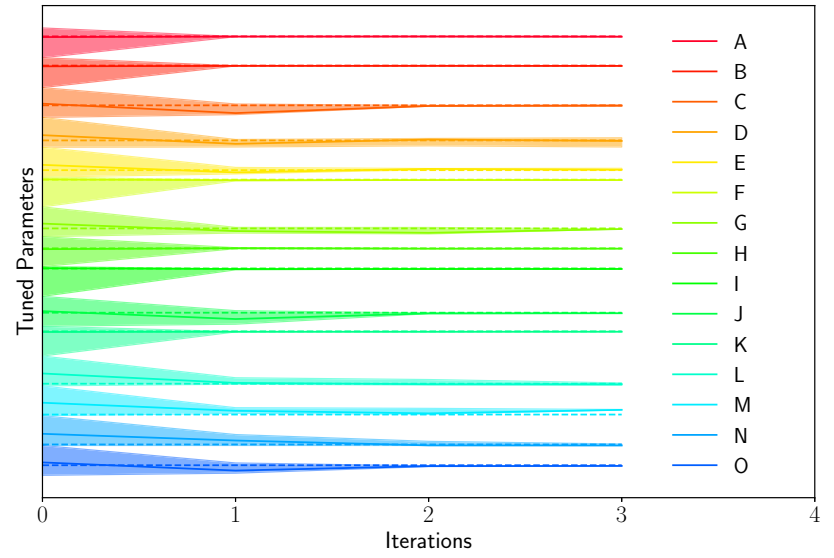

Fig. 4 Iterated tuning to polynomial pseudo data using the Autotunes method

out of the full set, with unit weights on all observables. In the second method, we choose the simultaneously tuned parameters based on physical motivation, but still use unit weights on all observables. Finally, we use the Autotunes method to divide the parameters into steps, and automatically set weights as described in Sect. 3.

The choice of parameters used in the physically motivated method is given in Table 1. The first step collects parameters that have a significant influence on many observables, combining shower and Pythia 8 string parameters. The second step gathers additional properties of the string model [33,34], focusing on the flavor composition. The last step then tunes the ratio of vector-to-pseudoscalar meson production.

The results of the three tuning approaches that aim to recover the Pythia 8 pseudo data parameters are shown in Fig. 5a-d. None of the approaches is capable of exactly recovering all of the original parameter values. This suggests that close-by points in parameter space are well suited to reproduce the pseudo data observable distributions. However, the iterated Autotunes method improves the agreement of the recovered parameters by avoiding large mismatches, e.g. in the StringFlav-mesonBvector parameter. In the physically motivated and random approaches, there is a certain chance that parameters are strongly constrained by observables that also depend on other parameters. If these are not identified and included in the same sub-tune, both parameters get constrained. Thus, the optimal configuration is not necessarily recovered, as can be observed by the seemingly fast convergence of the random method. Figure 5a shows that some parameters are constrained rather quickly, not recovering the original value. By iteratively identifying such sets of parameters, the Autotunes method attempts to avoid these mismatches. On the other side, Fig. 5b indicates that the fixed physically motivated combinations lead to a rather slow convergence of parameters. Overall, it is difficult to assess the tune quality from Fig. 5a to c.
Figure $5 \mathrm{~d}$ shows the summed, squared and normalized deviation of the recovered to the true parameter values. Each approach is performed three times to access the stability of the results. The random approach uses random combinations of parameters for the tuning steps, so we see a wide spread of results. The iterative tuning using our fixed physically motivated parameter choice is more reliable, showing a lower spread and better results. The Autotunes method leads to the best agreement with the original parameters. More stable results in the physically motivated and the Autotunes method could be achieved by using higher statistics for both the event generation and the sampling. We see that in the physically motivated and the Autotunes approach, a second tuning iteration affects the results, mostly - but not necessarily - improving the parameter agreement. Further iterations have a minor impact.

\section{Results}

We use the Autotunes framework to perform five distinct tunes to LEP observables. We tuned to a rather inclusive list of analyses ${ }^{9}$ available within the Rivet framework for the collider energy at the Z-Pole. To this point we do not weight the LEP observables, but make use of the sub-tune weights described in Sect. 3.2. ${ }^{10}$ The tunes make use of the default hadronisation models of the event generators Herwig 7 and Pythia 8. We further present a new tune of the Herwig 7 event generator interfaced to the Pythia 8 string hadronisation model. The details of the simulations can be found in the following sections. The results are presented in Table 2 and Table 3, listing default values, tuning ranges of the parameters, as well as the tuning results using the Autotunes method.

\footnotetext{
9 ALEPH-1991-S2435284 [36], ALEPH-1995-I382179 [37], ALEPH-1996-S3486095 [17], ALEPH-1999-S4193598 [38], ALEPH-2001-S4656318 [39], ALEPH-2002-S4823664 [40], ALEPH-2004-S5765862 [41], DELPHI-1991-I301657 [42], DELPHI-1995-S3137023 [43], DELPHI-1996-S3430090 [19], DELPHI-1999-S3960137 [44], DELPHI-2011-I890503 [45], JADEOPAL-2000-S4300807 [46], L3-1992-I336180 [47], L3-2004-I652683 [48], OPAL-1992-I321190 [49], OPAL-1993-I342766 [50], OPAL-1994-S2927284 [51], OPAL-1995-S3198391 [52], OPAL-1996-S3257789 [53], OPAL-1997-S3396100 [54], OPAL-1997-S3608263 [55], OPAL-1998-S3702294 [56], OPAL-1998-S3749908 [57], OPAL-1998-S3780481 [58], OPAL-2000-S4418603 [59], OPAL-2003-I599181 [60], OPAL-2004-I648738 [61], OPAL-2004-S6132243 [62], PDG-HADRON-MULTIPLICITIES [63], PDG-HADRON-MULTIPLICITIES-RATIOS [63], SLD-1996-S3398250 [64], SLD-1999-S3743934 [65], SLD-2002-S4869273 [66], SLD-2004-S5693039 [67].

${ }^{10}$ Additional weighting with knowledge of perturbative stability or known misinterpretation of experimental errors may be subject to future work.
} 
Table 1 Parameters chosen to be tuned simultaneously in the physics motivated tuning approach

\begin{tabular}{lll}
\hline Step 1 & Step 2 & Step 3 \\
\hline TimeShower:alphaSvalue & StringFlav:probStoUD & StringFlav:mesonUDvector \\
TimeShower:pTmin & StringFlav:probQQtoQ & StringFlav:mesonSvector \\
StringZ:aLund & StringFlav:probSQtoQQ & StringFlav:mesonCvector \\
StringZ:bLund & StringFlav:probQQ1toQQ0 & StringFlav:mesonBvector \\
StringPT:Sigma & StringFlav:etaSup & \\
StringZ:aExtraSQuark & StringFlav:etaPrimeSup & \\
StringZ:aExtraDiquark & StringFlav:popcornRate & \\
\hline
\end{tabular}

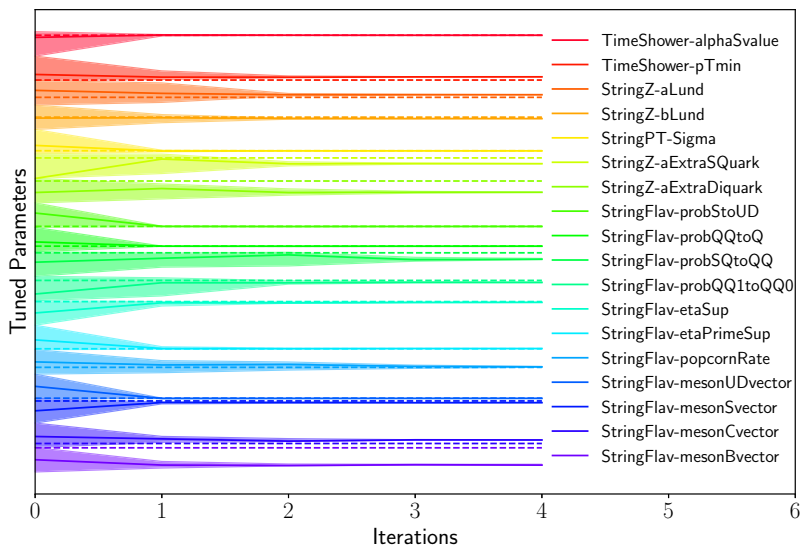

(b) Iterated Pythia 8 pseudo data tune with physically motivated choice of parameters.

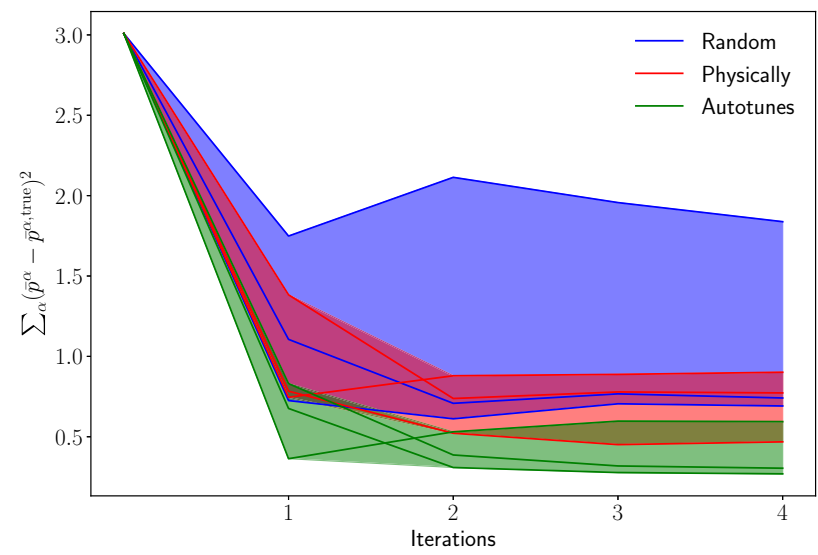

(d) Comparison in summed deviation from true parameters, each normalized to a range between 0 and 1 . Iterating the Autotunes approach leads to better agreement with initially chosen parameters.

bands are given by $80 \%$ of the best fit values in the Professor runcombinations and an additional 20\% margin. In Fig. 5d we compare the the summed deviation for three distinct tunes for the random, physically motivated and Autotunes method 


\subsection{Retuning of Pythia 8}

The tune of Pythia 8.235 is performed by using LEP data. We use Pythia 8's standard configuration as described in the manual, including a one-loop running of $\alpha_{\mathrm{S}}$ in the parton shower. The tuned parameters, initial ranges and tune results are given in Table 2 in Appendix B.

The given ranges on the tune results, obtained from the variation of the optimal tune in different run combinations, can be interpreted as a measure of the stability of the best tune. A wide range suggests that different configurations give tunes of similar $\chi^{2}$. The extraction of the strong coupling $\alpha_{S}$ is the most stable result in the tune. The modification of the longitudinal lightcone fraction distribution in the string fragmentation model for strange quarks (StringZ:aExtraSQuark) is very loosely constrained, suggesting that the data that is employed in the tune is not suitable to extract this parameter.

We tune 18 parameters in three sets of six parameters each. In the Pythia 8 tune, the parton shower cutoff pTmin is surprisingly loosely constrained. Checking the combinations of parameters that the Autotunes method chooses, we note that pTmin is found to be correlated with the string fragmentation parameters aLund and bLund in every iteration, which are also rather loosely constrained. This suggests that different choices for these three parameters can provide tunes of similar $\chi^{2}$.

\subsection{Retuning of Herwig 7}

As another real life example we tune the Herwig 7 event generator to LEP data. Here the tune is based upon version Herwig 7.1.4 and ThePEG 2.1.4. We perform two tunes - cluster and string model - for both showers, the QTilde shower [68] and the dipole shower [69]. For the presented tunes we do not employ the CMW scheme [70], but keep the $\alpha_{S}\left(M_{Z}\right)$ value a free parameter. This results in the enhanced value compared to the world average [71].

\subsubsection{Tuning Herwig 7 with cluster model}

We retune the cluster-model with a 22 dimensional parameter space. Here, we require tree sub-tunes and performed four iterations. The results are listed in Appendix B. Comparing the results, we note that the method is in general able to find values outside of the given initial parameter ranges, see e.g. the $\alpha_{S}\left(M_{Z}\right)$ or the nominal $b$-mass. This can be caused by Professor interpolation outside the given bounds or in the determination of the new ranges for the next iteration. Apart from the parameters that influence the cluster fission process of heavy clusters involving charm quarks (ClPowCharm and PSplitCharm), the parameters are comparable between the two shower models. Further in the cluster-model, the fission parameters are correlated. It is reasonable to assume possible local minima in the $\chi^{2}$ measure.

\subsubsection{Tuning Herwig 7 with Pythia 8 Strings}

The usual setup of the event generators are genuinely welltuned and even though the tests of Sect. 4 allow the conclusion that relatively arbitrary starting points lead to similar results, ignoring the previous knowledge completely seems undesirable. To create a real live example and further allow useful future studies we employed the fact that the $\mathrm{C}++$ version of the Ariadne shower but also the Herwig 7 event generator is based on ThePEG. Furthermore, with minor modifications, the unpublished interface between ThePEG and Pythia 8 (called TheP8I, written by L. Lönnblad), allowed the internal use of Pythia 8-stings with Herwig 7 events. ${ }^{11}$ Since no tuning for this setup was attempted before the starting conditions needed to be chosen with less bias compared to the other results of this section.

When we compare the values received for the Herwig 7 showers to the Pythia 8 shower, we note a comparably large value for the Pythia $8 \alpha_{\text {S }}$ value. In contrast, the cutoff in the transverse momentum in Pythia 8 is rather small. The reason for this contradicting behaviour ${ }^{12}$ can be found in the order at which the two codes evaluate the running of the strong coupling. While Herwig 7 chooses an NLO running, Pythia 8 evolves $\alpha_{\mathrm{S}}$ with LO running, and therefore suppresses the radiation for low energies. Even though the shower models are rather different, the difference in the response in the best fit values of the parameters are moderate. Less constrained parameters like the popcornRate, which influences part of the baryon production or the additional strange quark parameter aExtraSQuark show a corresponding large uncertainty. It can be concluded that the data used for tuning is hardly constraining these parameters.

\section{Conclusion and outlook}

We presented an algorithm that allows a semi-automatic Monte Carlo Event generator tuning of high dimensional parameter space. Here, we motivated and described how the parameter space can be split into sub-spaces, based on the projections to and variations in the observable space. We then assigned increased weights when we perform the sub-

\footnotetext{
${ }^{11}$ For the string model to be used with Herwig 7 the reshuffling to constituent masses was switched off. This modification will be available in the next Herwig 7 version. However, the underlying event modelling and needed colour reconnection is not yet supported and needs to be improved for the simulation of hadron collisions.

12 Observables like the number of charged particles are both likely to be modified in the same direction with an increased coupling and a decreased evolution cutoff.
} 
tunes, such that influential observables are highlighted. It is then possible to use the output of any tune step as starting conditions for next steps. Therefore the procedure is iterative. In ideal conditions, we performed tests to check that the algorithm finds correlated parameters and showed in realistic environment that pseudo data could be reproduced better by the algorithm than by random or physically motivated tunes. As real life examples we tuned the Pythia 8 and Herwig 7 showers with their standard hadronisations models and modified the Herwig 7 generator to allow consistent hadronisation with the Pythia 8's Lund String model.

The method allows to perform tuning with far less human interaction. It also allows different models to be tuned with a similar bias. Such tunes can then be used to identify mismodelling, with the assurance that the origin of the difference in data description is less likely part of a better or worse tuning.

At the current stage we did not assign weights or uncertainties other than the sub-tune weights and the uncertainties given by the experimental collaborations. We note that the difference between higher multiplicity merged simulations to the pure parton shower simulations can serve as an excellent reduction weight to suppress observables influenced by higher order calculations. However, the investigation of such procedures goes beyond the scope of this paper and will be subject to future work. Further, we did not address the third point of the mentioned restrictions in Sect. 2.1 that describes over-represented data. We postpone such studies, that include clustering of slope-vectors to reduce such an influence, to future work.

Acknowledgements We would like to thank Leif Lönnblad, Stefan Prestel and Holger Schulz for valuable discussions on the topic. We also thank Stefan Prestel and Malin Sjödahl for useful comments on the manuscript. This work has received funding from the European Union's Horizon 2020 research and innovation programme as part of the Marie Skłodowska-Curie Innovative Training Network MCnetITN3 (Grant agreement no. 722104). This project has also received funding from the European Research Council (ERC) under the European Union's Horizon 2020 research and innovation programme, grant agreement No 668679 .

Data Availability Statement This manuscript has no associated data or the data will not be deposited. [Author's comment: For this work, no data was measured that would justify additional material.]

Open Access This article is licensed under a Creative Commons Attribution 4.0 International License, which permits use, sharing, adaptation, distribution and reproduction in any medium or format, as long as you give appropriate credit to the original author(s) and the source, provide a link to the Creative Commons licence, and indicate if changes were made. The images or other third party material in this article are included in the article's Creative Commons licence, unless indicated otherwise in a credit line to the material. If material is not included in the article's Creative Commons licence and your intended use is not permitted by statutory regulation or exceeds the permitted use, you will need to obtain permission directly from the copyright holder. To view a copy of this licence, visit http://creativecomm ons.org/licenses/by/4.0/.

Funded by SCOAP ${ }^{3}$.

\section{Appendix A: Range dependence}

The algorithm to split the dimensions and to assign weights to sub-tunes is constructed such that correlations should still be found when the parameter ranges are varied. This is not always possible if the parameter ranges are strongly modified. It is possible that the slope vectors, that are evaluated by averaging over the full $n-1$ (other) dimensions, are modified by the newly defined initial ranges. It is even possible that the range of other parameters influence the slope as the spread modifies the normalisation. In order to show such behaviour (and also to illustrate the weight distributions), we choose three different setups for the event generator Herwig 7. We choose $d=4$ and try to split the dimensions in half. Here we choose the parameters and initial ranges as,

\begin{tabular}{llll}
\hline Parameter & Setup 1 & Setup 2 & Setup 3 \\
\hline$\alpha_{\mathrm{S}}\left(M_{\mathrm{Z}}\right)$ & $0.12-0.13$ & $0.124-0.126$ & $0.12-0.13$ \\
$C l_{\max }^{\text {light }}$ & $2.0-3.0$ & $2.4-2.6$ & $2.4-2.6$ \\
$p_{\min }^{T}$ & $0.7-0.9$ & $0.7-0.9$ & $0.78-0.82$ \\
$g_{\mathrm{CM}}$ & $0.7-0.9$ & $0.7-0.9$ & $0.7-0.9$ \\
\hline
\end{tabular}

The result for the parameter grouping and the weight distributions are depicted in Fig. 6. While the algorithm to split the parameter space in setup 1 and setup 2 such that $C l_{\max }^{\text {light }}$ and $p_{\min }^{T}$ should be tuned in the first step and then $\alpha_{\mathrm{S}}\left(M_{\mathrm{Z}}\right)$ and $g_{\mathrm{CM}}{ }^{13}$ in a second step, the modification to the initial ranges has the effect that the algorithm favours the pairing $\left(C l_{\max }^{\text {light }}, g_{\mathrm{CM}}\right)$ and $\left(\alpha_{\mathrm{S}}, p_{\min }^{T}\right)$ for steps 1 and 2 for setup 3 .

While it is possible that by changing the initial ranges the pairing flips and other parameter groups are found, the fact that neighbouring bins have a similar behaviour supports the concept of meaningful weight distributions. It would be possible to correlate neighbouring bins or introduce a smoothing algorithm to make the weights more stable but such a modification can be introduced once issues with the current algorithm appear.

In principle, it is possible to visualize for each parameter the weights of the sub-tune choice that we want. This choice can help to identify observables that are influential for individual parameters, and give insights in unexpected behaviours. Already from the weight distributions shown in Fig. 6, we can deduce that $p_{\min }^{T}$ is of great importance for the transverse momentum out-of-plane, see upper left panel. Further modifications of the constituent mass of the gluon $g_{\mathrm{CM}}$ will influence the difference in the hemisphere masses, see lower right panel.

\footnotetext{
${ }^{13} g_{\mathrm{CM}}$ is the parameter for the constituent mass of the gluon.
} 

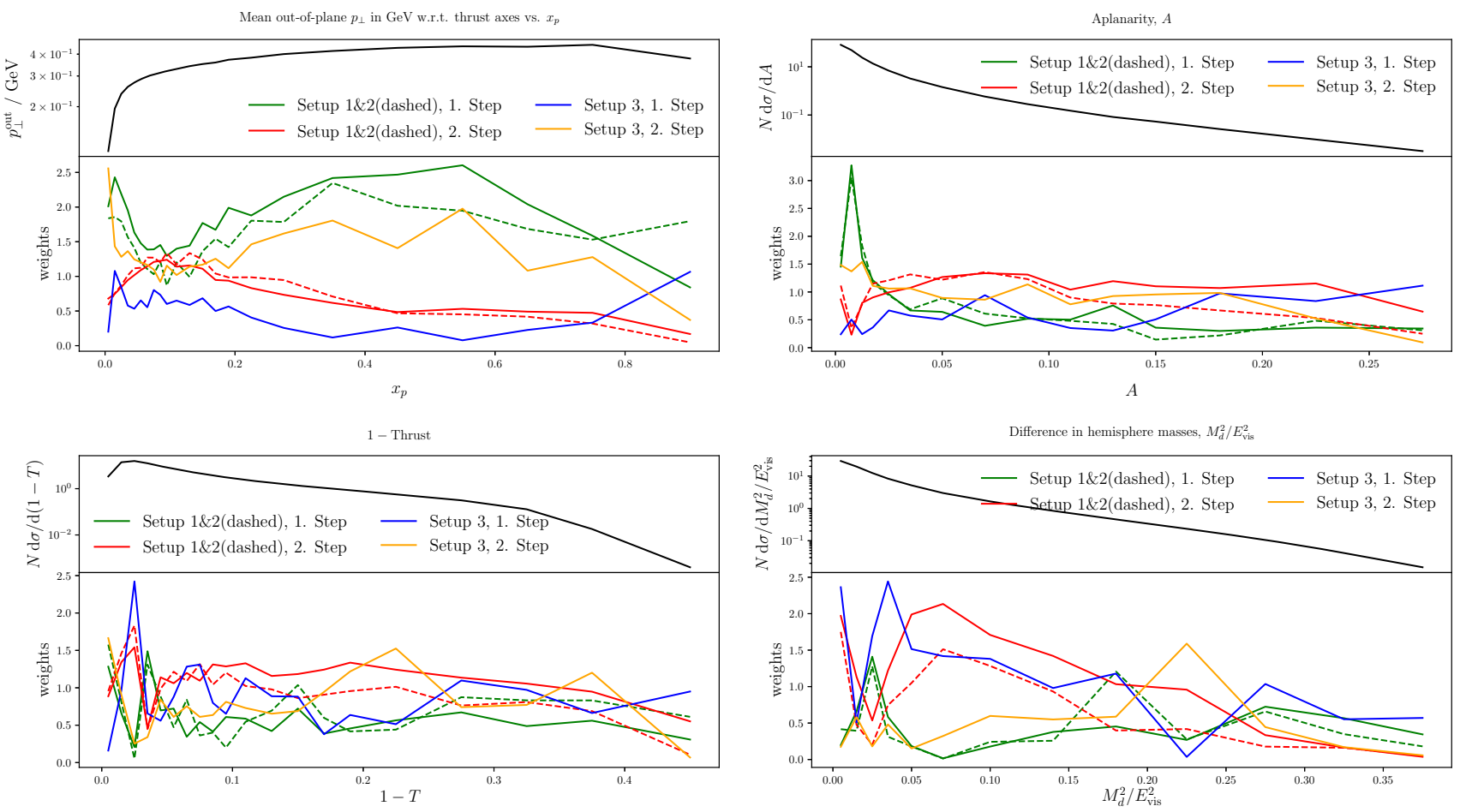

Fig. 6 Weight distributions for subsets of parameter pairs as described in Appendix A. The upper panels show the measured data points and the lower panels show the weights assigned. A clear distinction between

more and less important sets is visible. The dashed lines correspond to Setup 2, which gives a same grouping of parameters as Setup 1

\section{Appendix B: Tune Results}

In Table 2 and Table 3 we list the results of the Herwig 7 and Pythia 8 tunes with the standard hadronisation. For Herwig 7 we also list a tune for the Lund string model. The results are discussed in Sect. 5 . 
Table 2 Tuned Pythia 8 parameters with default values, initial ranges for the tune, and the Autotunes result. See $[7,8]$ for details on the parameters. $(\mathrm{SF}=$ StringFlav, $\mathrm{SZ}=$ StringZ). The errors given are determined by the method described in Sects. 3.4 and 3.3 and must not be confused with either theoretical or experimental uncertainties
Table 3 Result of the retuning of the Herwig 7 event generator employing the default hadronisation model (cluster model). The 22 dimensional parameter space was tuned with three sub-tunes and three iterations. Shown are the default values (corresponding to the Qtilde shower tune) as well as the parameter range and the tuning result for both showers. The errors given are determined by the method described in Sects. 3.4 and 3.3 and must not be confused with neither theoretical not experimental uncertainties

\begin{tabular}{llllll}
\hline Parameter & Def. & Range & Pythia 8 tune & H7+ + +Str. & H7+Dip.+Str. \\
\hline alphaSvalue & 0.1365 & $0.125-0.14$ & $0.13699_{-0.00057}^{+0.00019}$ & $0.1289_{-0.0004}^{+0.0011}$ & $0.13229_{-0.00015}^{+0.00083}$ \\
pTmin & 0.5 & $0.4-0.8$ & $0.49_{-0.16}^{+0.05}$ & $0.993_{-0.004}^{+0.010}$ & $0.990_{-0.004}^{+0.020}$ \\
SZ-aLund & 0.68 & $0.5-0.8$ & $0.71_{-0.24}^{+0.07}$ & $0.60_{-0.19}^{+0.13}$ & $0.84_{-0.16}^{+0.03}$ \\
SZ-bLund & 0.98 & $0.7-1.3$ & $1.11_{-0.23}^{+0.11}$ & $0.78_{-0.19}^{+0.18}$ & $1.00_{-0.20}^{+0.04}$ \\
StringPT-Sigma & 0.335 & $0.3-0.4$ & $0.3011_{-0.0010}^{+0.0020}$ & $0.3008_{-0.0022}^{+0.0006}$ & $0.29876_{-0.00022}^{+0.00122}$ \\
SZ-aExtraSQuark & 0.0 & $0.0-0.5$ & $0.04_{-0.05}^{+0.60}$ & $0.08_{-0.08}^{+0.26}$ & $0.22_{-0.22}^{+0.37}$ \\
SZ-aExtraDiquark & 0.97 & $0.8-1.2$ & $1.19_{-0.18}^{+0.06}$ & $1.1_{-0.6}^{+0.3}$ & $1.02_{-0.18}^{+0.38}$ \\
SF-probStoUD & 0.217 & $0.1-0.3$ & $0.196_{-0.004}^{+0.010}$ & $0.2186_{-0.0103}^{+0.0018}$ & $0.1979_{-0.0066}^{+0.0016}$ \\
SF-probQQtoQ & 0.081 & $0.0-0.2$ & $0.0828_{-0.0024}^{+0.0011}$ & $0.0821_{-0.0032}^{+0.0010}$ & $0.0856_{-0.0039}^{+0.0007}$ \\
SF-probSQtoQQ & 0.915 & $0.8-1.0$ & $0.98_{-0.05}^{+0.09}$ & $0.748_{-0.029}^{+0.091}$ & $0.797_{-0.009}^{+0.004}$ \\
SF-probQQ1toQQ0 & 0.0275 & $0.0-0.1$ & $0.033_{-0.011}^{+0.003}$ & $0.024_{-0.006}^{+0.008}$ & $0.023_{-0.003}^{+0.007}$ \\
SF-etaSup & 0.6 & $0.4-0.8$ & $0.644_{-0.018}^{+0.034}$ & $0.800_{-0.036}^{+0.012}$ & $0.7976_{-0.0068}^{+0.0018}$ \\
SF-etaPrimeSup & 0.12 & $0.0-0.3$ & $0.1095_{-0.0016}^{+0.0054}$ & $0.1027_{-0.0022}^{+0.0115}$ & $0.100_{-0.008}^{+0.017}$ \\
SF-popcornRate & 0.5 & $0.4-0.6$ & $0.31_{-0.05}^{+0.27}$ & $0.63_{-0.36}^{+0.12}$ & $0.51_{-0.17}^{+0.08}$ \\
SF-mesonUDvector & 0.5 & $0.3-0.7$ & $0.527_{-0.023}^{+0.027}$ & $0.459_{-0.008}^{+0.031}$ & $0.473_{-0.047}^{+0.009}$ \\
SF-mesonSvector & 0.55 & $0.35-0.75$ & $0.53_{-0.08}^{+0.07}$ & $0.55_{-0.05}^{+0.07}$ & $0.581_{-0.044}^{+0.018}$ \\
SF-mesonCvector & 0.88 & $0.7-1.1$ & $0.874_{-0.024}^{+0.021}$ & $1.10_{-0.22}^{+0.008}$ & $0.72_{-0.08}^{+0.13}$ \\
SF-mesonBvector & 2.2 & $2.0-2.4$ & $2.24_{-0.20}^{+0.16}$ & $2.34_{-0.50}^{+0.09}$ & $2.31_{-0.44}^{+0.27}$ \\
\hline
\end{tabular}

\begin{tabular}{lllll}
\hline Parameter & Def. & Range & H7+Dip.+Cluster & H7+ + + Cluster \\
\hline alphaS & 0.126234 & $0.12-0.13$ & $0.13008_{-0.00061}^{+0.00013}$ & $0.12455_{-0.00118}^{+0.00020}$ \\
gConstituentMass & 0.95 & $0.7-1.1$ & $0.83_{-0.07}^{+0.16}$ & $1.0045_{-0.0006}^{+0.0028}$ \\
EMpTmin & 1.2228 & $0.8-1.4$ & $1.204_{-0.033}^{+0.010}$ & $1.068_{-0.020}^{+0.004}$ \\
SPpTmin & 1.2228 & $0.8-1.4$ & $1.204_{-0.033}^{+0.010}$ & $1.22_{-0.74}^{+0.27}$ \\
bNominalMass & 4.2 & $4.0-4.7$ & $4.76_{-0.21}^{+0.04}$ & $4.2_{-0.5}^{+0.9}$ \\
bConstituentMass & 5. & $4.0-4.7$ & $4.01_{-0.13}^{+0.22}$ & $4.03_{-0.15}^{+0.17}$ \\
DecWt & 0.62 & $0.5-0.9$ & $0.59_{-0.05}^{+0.10}$ & $0.61_{-0.04}^{+0.05}$ \\
SngWt & 0.74 & $0.5-0.9$ & $0.86_{-0.11}^{+0.18}$ & $0.80_{-0.37}^{+0.14}$ \\
ClSmrLight & 0.78 & $0.5-1.0$ & $0.59_{-0.08}^{+0.05}$ & $0.437_{-0.013}^{+0.041}$ \\
ClSmrCharm & 0. & $0.0-0.2$ & $0.24_{-0.21}^{+0.04}$ & $0.18_{-0.18}^{+0.04}$ \\
ClSmrBottom & 0.0204 & $0.0-0.1$ & $0.100_{-0.044}^{+0.019}$ & $0.088_{-0.033}^{+0.018}$ \\
ClMaxLight & 3.00254 & $3.0-5.0$ & $3.18_{-0.22}^{+0.11}$ & $3.13_{-0.14}^{+0.08}$ \\
ClMaxCharm & 3.63822 & $3.0-5.0$ & $3.34_{-0.07}^{+0.25}$ & $3.68_{-0.07}^{+0.28}$ \\
ClMaxBottom & 3.911 & $3.0-5.0$ & $4.4_{-0.6}^{+1.6}$ & $4.9_{-2.9}^{+0.9}$ \\
ClPowLight & 1.42426 & $1.0-1.8$ & $1.85_{-0.58}^{+0.24}$ & $1.36_{-0.03}^{+0.15}$ \\
ClPowCharm & 2.33186 & $1.5-3.0$ & $1.89_{-0.29}^{+1.71}$ & $3.1_{-1.5}^{+0.4}$ \\
ClPowBottom & 0.6375 & $0.4-0.8$ & $0.638_{-0.018}^{+0.104}$ & $0.80_{-0.23}^{+0.04}$ \\
PSplitLight & 0.847541 & $0.0-1.5$ & $0.8747_{-0.0007}^{+0.0041}$ & $0.935_{-0.018}^{+0.035}$ \\
PSplitCharm & 1.23399 & $0.0-1.5$ & $0.637_{-0.028}^{+0.164}$ & $1.20_{-0.66}^{+0.11}$ \\
PSplitBottom & 0.5306 & $0.0-1.5$ & $0.599_{-0.113}^{+0.019}$ & $0.69_{-0.12}^{+0.12}$ \\
SingleHadronLimitCharm & 0.0 & $0.0-0.5$ & $0.0015_{-0.0015}^{+0.0156}$ & $0.0012_{-0.0012}^{+0.0061}$ \\
SingleHadronLimitBottom & 0.0 & $0.0-0.5$ & $0.08_{-0.09}^{+0.12}$ & $0.015_{-0.016}^{+0.009}$ \\
\hline & & &
\end{tabular}




\section{References}

1. A. Buckley et al., Phys. Rep. 504, 145 (2011). arXiv:1101.2599

2. M. Bähr et al., Eur. Phys. J. C 58, 639 (2008). arXiv:0803.0883

3. J. Bellm et al., Eur. Phys. J. C 76, 196 (2016). arXiv:1512.01178

4. S. Platzer, S. Gieseke, Eur. Phys. J. C 72, 2187 (2012). arXiv: 1109.6256

5. J. Bellm et al., (2017). arXiv: 1705.06919

6. T. Gleisberg, S. Höche, F. Krauss, A. Schalicke, S. Schumann, J.-C. Winter, JHEP 02, 056 (2004). arXiv:hep-ph/0311263

7. T. Sjöstrand, S. Mrenna, P.Z. Skands, JHEP 05, 026 (2006). arXiv:hep-ph/0603175

8. T. Sjöstrand, S. Ask, J.R. Christiansen, R. Corke, N. Desai, P. Ilten, S. Mrenna, S. Prestel, C.O. Rasmussen, P.Z. Skands, Comput. Phys. Commun. 191, 159 (2015). arXiv: 1410.3012

9. D. Reichelt, P. Richardson, A. Siodmok, Eur. Phys. J. C 77, 876 (2017). arXiv: 1708.01491

10. S. Gieseke, P. Kirchgaeßer, S. Plätzer, Eur. Phys. J. C 78, 99 (2018). arXiv: 1710.10906

11. S. Höche, S. Prestel, Phys. Rev. D 96, 074017 (2017). arXiv: 1705.00742

12. S. Höche, F. Krauss, S. Prestel, JHEP 10, 093 (2017). arXiv:1705.00982

13. J. Bellm, Eur. Phys. J. C 78, 601 (2018). arXiv: 1801.06113

14. C.B. Duncan, P. Kirchgaeßer, Eur. Phys. J. C 79, 61 (2019). arXiv:1811.10336

15. F. Dulat, S. Höche, S. Prestel, Phys. Rev. D 98, 074013 (2018). arXiv: 1805.03757

16. G. Bewick, S. Ferrario Ravasio, P. Richardson, M.H. Seymour (2019). arXiv: 1904.11866

17. R. Barate et al. (ALEPH), Phys. Rep. 294, 1 (1998)

18. K. Hamacher, M. Weierstall (DELPHI) (1995). arXiv:hep-ex/9511011

19. P. Abreu et al. (DELPHI), Z. Phys. C 73, 11 (1996)

20. P. Z. Skands, In: Proceedings, 1st International Workshop on Multiple Partonic Interactions at the LHC (MPI08): Perugia, Italy, October 27-31, 2008 (2009), pp. 284-297. arXiv:0905.3418, http://lss. fnal.gov/cgi-bin/find_paper.pl?conf-09-113

21. A. Buckley, H. Hoeth, H. Lacker, H. Schulz, J.E. von Seggern, Eur. Phys. J. C 65, 331 (2010). arXiv:0907.2973

22. P. Skands, S. Carrazza, J. Rojo, Eur. Phys. J. C 74, 3024 (2014). arXiv: 1404.5630

23. V. Khachatryan et al. (CMS), Eur. Phys. J. C 76, 155 (2016). arXiv: 1512.00815

24. P. Ilten, M. Williams, Y. Yang, JINST 12, P04028 (2017). arXiv: 1610.08328

25. A. Buckley, H. Schulz, Adv. Ser. Direct. High Energy Phys. 29, 281 (2018). arXiv:1806.11182

26. J. Bellm, S. Plätzer, P. Richardson, A. Siódmok, S. Webster, Phys. Rev. D 94, 034028 (2016). arXiv: 1605.08256

27. S. Mrenna, P. Skands, Phys. Rev. D 94, 074005 (2016). arXiv: 1605.08352

28. E. Bothmann, M. Schönherr, S. Schumann, Eur. Phys. J. C 76, 590 (2016). arXiv: 1606.08753

29. E. Bothmann, L. Debbio, JHEP 01, 033 (2019). arXiv:1808.07802

30. A. Andreassen, B. Nachman (2019). arxiv:1907.08209

31. E. Maguire, L. Heinrich, G. Watt, J. Phys. Conf. Ser. 898, 102006 (2017). arXiv: 1704.05473

32. A. Buckley, J. Butterworth, L. Lonnblad, D. Grellscheid, H. Hoeth, J. Monk, H. Schulz, F. Siegert, Comput. Phys. Commun. 184, 2803 (2013). arXiv:1003.0694

33. B. Andersson, G. Gustafson, G. Ingelman, T. Sjöstrand, Phys. Rep. 97, 31 (1983)

34. T. Sjöstrand, Nucl. Phys. B 248, 469 (1984)
35. G. Aad et al., (ATLAS). Eur. Phys. J. C 76, 199 (2016). arXiv: 1508.00848

36. D. Decamp et al. (ALEPH), Phys. Lett. B 273, 181 (1991)

37. D. Buskulic et al. (ALEPH), Z. Phys. C 66, 355 (1995)

38. R. Barate et al. (ALEPH), Eur. Phys. J. C 16, 597 (2000) arXiv:hep-ex/9909032

39. A. Heister et al. (ALEPH), Phys. Lett. B 512, 30 (2001). arXiv:hep-ex/0106051

40. A. Heister et al. (ALEPH), Phys. Lett. B 528, 19 (2002). arXiv:hep-ex/0201012

41. A. Heister et al. (ALEPH), Eur. Phys. J. C 35, 457 (2004)

42. P. Abreu et al. (DELPHI), Z. Phys. C 50, 185 (1991)

43. P. Abreu et al. (DELPHI), Z. Phys. C 67, 543 (1995)

44. P. Abreu et al. (DELPHI), Phys. Lett. B 449, 364 (1999)

45. J. Abdallah et al. (DELPHI), Eur. Phys. J. C 71, 1557 (2011). arXiv: 1102.4748

46. P. Pfeifenschneider et al. (JADE, OPAL), Eur. Phys. J. C 17, 19 (2000). arXiv:hep-ex/0001055

47. O. Adriani et al. (L3), Phys. Lett. B286, 403 (1992)

48. P. Achard et al., (L3). Phys. Rep. 399, 71 (2004). arXiv:hep-ex/0406049

49. P.D. Acton et al. (OPAL), Z. Phys. C 53, 539 (1992)

50. P.D. Acton et al. (OPAL), Phys. Lett. B 305, 407 (1993)

51. R. Akers et al. (OPAL), Z. Phys. C 63, 181 (1994)

52. G. Alexander et al. (OPAL), Phys. Lett. B 358, 162 (1995)

53. G. Alexander et al. (OPAL), Z. Phys. C 70, 197 (1996)

54. G. Alexander et al. (OPAL), Z. Phys. C 73, 569 (1997)

55. K. Ackerstaff et al. (OPAL), Phys. Lett. B 412, 210 (1997). arXiv:hep-ex/9708022

56. K. Ackerstaff et al. (OPAL), Eur. Phys. J. C 4, 19 (1998a). arXiv:hep-ex/9802013

57. K. Ackerstaff et al. (OPAL), Eur. Phys. J. C 5, 411 (1998b). arXiv:hep-ex/9805011

58. K. Ackerstaff et al. (OPAL), Eur. Phys. J. C 7, 369 (1999). arXiv:hep-ex/9807004

59. G. Abbiendi et al. (OPAL), Eur. Phys. J. C 17, 373 (2000). arXiv:hep-ex/0007017

60. G. Abbiendi et al. (OPAL), Eur. Phys. J. C 29, 463 (2003). arXiv:hep-ex/0210031

61. G. Abbiendi et al. (OPAL), Eur. Phys. J. C 37, 25 (2004). arXiv:hep-ex/0404026

62. G. Abbiendi et al., (OPAL). Eur. Phys. J. C 40, 287 (2005). arXiv:hep-ex/0503051

63. C. Amsler et al. (Particle Data Group), Phys. Lett. B 667, 1 (2008)

64. K. Abe et al., (SLD). Phys. Lett. B 386, 475 (1996). arXiv:hep-ex/9608008

65. K. Abe et al. (SLD), Phys. Rev. D 59, 052001 (1999). arXiv:hep-ex/9805029

66. K. Abe et al. (SLD), Phys. Rev. D65, 092006 (2002), [Erratum: Phys. Rev.D66,079905(2002)], arXiv:hep-ex/0202031

67. K. Abe et al., (SLD). Phys. Rev. D 69, 072003 (2004). arXiv:hep-ex/0310017

68. S. Gieseke, P. Stephens, B. Webber, JHEP 12, 045 (2003). arXiv:hep-ph/0310083

69. S. Platzer, S. Gieseke, JHEP 01, 024 (2011). arXiv:0909.5593

70. S. Catani, B.R. Webber, G. Marchesini, Nucl. Phys. B 349, 635 (1991)

71. M. Tanabashi et al., (Particle Data Group). Phys. Rev. D 98, 030001 (2018) 